\title{
La situación sociosanitaria de los refugiados civiles españoles en Francia, marzo-junio de 1939
}

\author{
Rubén Mirón-González (*) y Carmen González-Canalejo (**) \\ ${ }^{*}$ ) orcid.org/0000-0003-4788-6083. Facultad de Medicina y Ciencias de la Salud. Universidad \\ de Alcalá. ruben.miron@uah.es \\ ${ }^{(* *}$ ) orcid.org/0000-0003-0843-1289. Facultad de Ciencias de la Salud. Universidad de Almería
}

Dynamis

[0211-9536] 2019; 39 (2): 429-452

http://dx.doi.org/10.30827/dynamis.v39i2.9844
Fecha de recepción: 30 de abril de 2018

Fecha de aceptación: 2 de julio de 2109

SUMARIO: 1.-Introducción. 2.-Campos de concentración y centros de alojamiento, una aproximación contextual. 3.-La llegada de las organizaciones de ayuda a los centros de alojamiento. 4. - Las condiciones de vida. 5.-La presión psicológica y el aislamiento social. 6.-La asistencia sanitaria. 7.-Los rincones blancos. 8.-Conclusiones.

RESUMEN: El artículo intenta aproximarse a la realidad sociosanitaria de más de 210 mil refugiados civiles que cruzaron la frontera pirenaica en el invierno de 1939. Este colectivo estuvo constituido principalmente por mujeres, niños y ancianos. A su llegada a Francia, la población civil fue reconducida hacia más de 1.500 centros de alojamiento. Nos encontramos ante espacios que se diferenciaron de los campos de concentración en lo que respecta a libertad y trato humano. Hemos podido acercarnos a esta realidad gracias a los informes emitidos por las delegadas de la Commission d'Aide aux Enfants Espagnols Refugies en France entre marzo y junio de 1939.

PALABRAS CLAVE: exilio, guerra civil española, refugiados, Francia, campos de concentración.

KEYWORDS: exile, Spanish civil war, refugees, France, concentration camps. 


\section{Introducción $(*)$}

Entre finales de enero y el 13 de febrero de 1939 atravesaron los Pirineos orientales cerca de medio millón de refugiados españoles huyendo del avance de las tropas franquistas sobre Cataluña. En primer lugar, Francia dejó pasar al colectivo comprendido por mujeres, niños y ancianos. Esto supuso la acogida de más de 210 mil refugiados, que se sumaron a los que ya había en Francia desde los inicios de la guerra civil española ${ }^{1}$. Estos refugiados fueron conducidos hacia el interior de Francia y asilados en más de 1.500 centros de alojamiento repartidos por gran parte de la geografía francesa. Junto al primer colectivo civil, también se permitió la entrada a más de 13 mil heridos y enfermos ${ }^{2}$. En último lugar, se accedió a la llegada de más de 220 mil militares de la retaguardia catalana ${ }^{3}$. Esto fue posible a partir del 5 y 6 de febrero de 1939, momento en el que el gobierno francés decidió abrir los principales puntos de acceso de Cerbère y Le-Perthus. Dichos militares fueron conducidos hacia las playas del Rosellón, donde se crearon improvisados campos de concentración ${ }^{4}$.

En esta investigación, vamos a seguir la pista del primer grupo de refugiados civiles que entraron en Francia. Desde que defendió la tesis doctoral Jeanine Sodigné-Loustau en $1995^{5}$, se ha incrementado el número de investigaciones que han analizado la recepción, el alojamiento y/o la vida cotidiana

(*) Investigación asociada al proyecto El exilio republicano andaluz de 1939 del Ministerio de la Presidencia (n. 321.1) entre los años 2010 y 2011, y al proyecto Acciones de socorro y tecnologías Médicas en emergencias humanitarias (1850-1950): agencias, agendas, espacios y representaciones del Ministerio de Ciencia, Innovación y Universidades (HAR2015-67723-P) entre los años 2016 y 2019.

1. Datos según el informe Valière de la Cámara de los Diputados en: Dreyfus-Armand, Geneviève. El exilio de los republicanos españoles en Francia. De la guerra civil a la muerte de Franco. Barcelona: Crítica; 2000, p. 53.

2. Mirón-González, Rubén; González-Canalejo, Carmen. La asistencia sanitaria a los heridos y enfermos del exilio republicano español en Francia: de la improvisación inicial a los campos de concentración (enero-septiembre 1939). Asclepio. 2018; 70 (2): 234-253.

3. Informe Valière en Dreyfus-Armand, n. 1.

4. Rafaneau-Boj, Marie Claude. Los campos de concentración de los refugiados españoles en Francia (1939-1945). Barcelona: Omega; 1995. Dreyfus-Armand, n. 1. Peschanski, Denis. La France des camps. L'internement, 1938-1946. Paris: Gallimard; 2002.

5. Sodigné-Loustau, Jeanine. L'immigration politique espagnole en région centre (Cher, Eure-et-Loir, Loir-et-Cher, Loiret) de 1936 à 1946. Paris: Université Paris 7; 1995. 
de los refugiados civiles en diferentes zonas geográficas de Francia ${ }^{6}$. De esta forma, nos encontramos ante una importante representación de trabajos que aportan una visión local o regional de lo que supuso la recepción de los exiliados españoles. Asimismo, dado que la gran mayoría de civiles que llegaron a Francia fueron mujeres, el examen de los testimonios femeninos ha resultado crucial para el análisis cualitativo del exilio civil ${ }^{7}$. Sin embargo, a pesar de los esfuerzos realizados en los últimos años, seguimos echando en falta estudios que analicen la realidad de los refugiados civiles bajo un punto de vista sociosanitario y con una mayor amplitud geográfica. Por todo ello, nos enfrentamos a una tarea compleja, a la que se suma la dificultad del acceso a las fuentes primarias debido a la diáspora que significó la «Retirada» de $1939^{8}$.

Ese mismo año, la administración pública francesa decidía encomendar la cuestión de los refugiados civiles a organizaciones humanitarias y comités de ayuda ${ }^{9}$. Una de las consecuencias de la llegada de las comisiones de ayuda internacional a los campos de concentración y a los centros de alojamiento civiles franceses, fue la visibilización de las condiciones en las que estaban viviendo estos españoles durante los primeros meses del éxodo de 1939. Una realidad que podemos conocer gracias a los informes emitidos por las delegadas de la Commission d'Aide aux Enfants Espagnols Refugies en France

6. Giraudier, Vincent; Mauran, Hervé; Sauvageon, Jean; Serre, Robert. Des indésirables: les camps d'internement et de travail dans l'Ardèche et la Drôme durant la Seconde Guerre mondiale. Valence: Peuple libre; 1999; Luis, Jean-Philippe. La comunidad española en Francia: la región de Auvergne. Hispania. 2002; 62/2 (211): 597-616; Alted, Alicia; Domergue, Lucienne (coords.). El exilio republicano español en Toulouse, 1939-1999. Madrid: UNED; 2003; Léger, Eva. Ejemplos de represión contra comunistas españoles y franceses en 1939 en el centro-oeste de Francia (departamento de la Haute-Vienne). Historia Actual Online. 2010; 22 (primavera): 77-83. Parello, Vincent. Des réfugiés espagnols de la guerre civile dans le département de l'Hérault (1937-1939). Saint-Estève: Presses Universitaires de Perpignan; 2010; Léger, Eva. Solidaridades y antifascismo. Las relaciones entre lemosines y españoles (1936-1945). Migraciones y Exilios. 2012; 13: 45-59.

7. Simón Porolli, Paula. Los campos de concentración franceses contados por las mujeres: aportes para la reflexión sobre la narrativa testimonial femenina. Laberintos. 2012; 14: 151-165.

8. Mirón-González, Rubén. Fuentes archivísticas para el estudio sanitario del exiliado español en el sur de Francia (1936-1945). En: Bellver Loizaga, Vicent; et al. Otras voces, otros ámbitos: los sujetos y su entorno. Nuevas perspectivas de la historia sociocultural. Valencia: Universitat de València; 2015, p. 208-211.

9. Colomina, Inmaculada. Fuera de los campos. Acciones de ayuda humanitaria para las mujeres españolas refugiadas en Francia. En: Alted Vigil, Alicia; Fernández Martínez, Dolores, eds. Tiempos de exilio y solidaridad: la Maternidad Suiza de Elna (1939-1944). Madrid: UNED; 2014, p. 89-101. 
$(\text { CAEERF })^{10}$. Además, se ha complementado la investigación con documentación de cinco archivos departamentales franceses ${ }^{11}$.

\section{Campos de concentración y centros de alojamiento, una aproximación contextual}

En los inicios de la guerra civil española, los refugiados españoles en Francia ocuparon diversos tipos de alojamientos. Entre 1936 y 1937, la mayoría de dichos centros tuvieron un carácter provisional y fueron de iniciativa privada, pero a partir de la «Retirada» de 1939, eso cambió. Rápidamente, se fueron creando los primeros «campos de concentración» franceses, un término que ha perdurado a lo largo del tiempo a través de los testimonios de los refugiados y que su uso entre historiadores sigue suscitando cierta polémica, especialmente entre franceses y españoles ${ }^{12}$.

\section{Tabla 1}

Terminología usada para hacer referencia a los distintos espacios destinados al alojamiento y asistencia de refugiados españoles en Francia (1936-1945).

\begin{tabular}{|l|l|}
\hline \multicolumn{2}{|c|}{ CORTO PLAZO } \\
\hline \multicolumn{1}{|c|}{ FRANCÉS } & \multicolumn{1}{c|}{ ESPAÑOL } \\
\hline - Postes de secours/sanitaires. & - Puestos de primeros auxilios/sanitarios. \\
- Centres de recueil/accueil. & - Centros de acogida/recepción. \\
- Postes/Camps de triage. & - Puestos/Campos de clasificación. \\
- Postes d'épouillage. & - Puestos de despiojamiento. \\
- Camps de circonstance. & - Campos de circunstancia. \\
\hline
\end{tabular}

10. Disponible en los Archives Nationales de Francia (ANF). Los informes de la CAEERF también han sido trabajados por la investigadora Maëlle Maugrendre, en su caso bajo un análisis de género y con Archivos Departamentales diferentes a este trabajo. Maugendre, Maëlle. Femmes en exil: Les refugiées espagnoles en France (1939-1942). Tours: Presses Universitaire François Rabelais; 2019.

11. Archives Départementales de l'Ariège (ADARI), de Aude (ADAUD), de Pyrénées-Orientales (ADPO), de Tarn (ADTA), de Tarn-et-Garonne (ADTG).

12. A modo de ejemplo, este debate surgió en el Colloque International de Carcassonne, celebrado el 4 de junio de 2004. Archives Départementales de l'Aude. Réfugiés espagnols dans l'Aude, 1939-1940. Actes du colloque international de Carcassonne (4 juin 2004). Carcassonne: Conseil Général de l'Aude; 2005. 


\begin{tabular}{|l|l|}
\hline \multicolumn{2}{|c|}{ MEDIO PLAZO } \\
\hline \multicolumn{1}{|c|}{ FRANCÉS } & \multicolumn{1}{c|}{ ESPANOL } \\
\hline - Camps/Centres de réception. & - Campos/Centros de recepción. \\
- Centres d'évacuation. & - Centros de evacuación. \\
- Camps/Comunes d'accueil provisoires. & - Campos/Municipios de acogida pro- \\
- Centres dé emigration. & visional. \\
- Camps-hôpitaux. & - Centros de emigración. \\
- Bateaux-hôpitaux. & - Campos hospitales. \\
\hline \multicolumn{1}{|c|}{ FRANCÉS } & LARGO hospitales. \\
\hline - Camps de réfugiés. & PAZO \\
\hline - Camps/Centres d'hérbeguement. & - Campos de refugiados. \\
- Camps de concentration. & - Campos de concentración. \\
- Camps de surveillance. & - Campos de internamiento. \\
- Camps d'étrangers. & - Campos de vigilancia. \\
- Camps militaires. & - Campos de extranjeros. \\
- Camps civiles. & - Campos militares. \\
- Centres de séjour surveillé. & - Campos civiles. \\
\hline
\end{tabular}

Fuente: Elaboración propia.

La utilización del término «centro de acogida»o «recepción» (accueil o recueil) por las autoridades francesas se remonta a 1937. Su planteamiento surge tras la «desbandá» de Málaga y la movilización masiva de refugiados hacia Francia ${ }^{13}$ (véase la terminología expuesta en la tabla 1). Los «centros de acogida» estuvieron pensados para el alojamiento de mujeres, niños y ancianos. Pronosticando la previsible afluencia de españoles, dichos centros tomaron medidas para el acondicionamiento de los posibles recién llegados. Por un lado, se prepararían camas para ancianos y niños, mientras que para el resto de los acogidos se aprovisionarían camastros de paja. Y por el otro, se dispuso la instalación de «centros de acogida» cerca de las fronteras y la creación de puestos fronterizos sanitarios (postes de secours) para la desparasitación (épouillage) y el triaje ${ }^{14}$.

13. Rubio, Javier. La emigración española en Francia. Esplugas de Llobregat; Ariel: 1974, p. 193.

14. En estos centros los refugiados pasaban un examen médico y se les vacunaba contra la viruela. Los heridos graves eran clasificados en los puestos de triaje y trasladados a los hospitales 
A mediados de 1937 comenzó a utilizarse el término «campo»o «centro de alojamiento» (hébergement). Se trataba de centros situados en el interior de Francia destinados a la población civil (mujeres, niños y ancianos) ${ }^{15}$. Respecto a la población militar, el prototipo de centro fue el de los «campos de circunstancia» creados en 1938. Éstos fueron los predecesores de los denominados «campos de concentración» ${ }^{16}$. La utilización del término «campo de concentración» por parte de las autoridades francesas se hizo latente a partir de la «Retirada» de 1939. A pesar de que las autoridades políticas evitaron usar dicho concepto en sus informes, a finales de enero de ese año, la prensa francesa ya lo empleaba en artículos ${ }^{17}$. Igualmente, Albert Sarraut, ministro del Interior francés, utilizó la expresión «campo de concentración» de forma abierta en un discurso pronunciado en la Cámara de los Diputados el 11 de marzo de $1939^{18}$.

El «universo concentracionario» ${ }^{19}$, poco a poco fue definiéndose a medida que avanzó la II Guerra Mundial. En consecuencia, en 1941 apareció la primera clasificación de campos por parte del Ministerio del Interior del Gobierno de Vichy. Tras dos años de experiencia, los campos se organizaron en tres categorías: «campos de internamiento» (internement), «campos de alojamiento» (hébergement) y «centros de emigración» (emigration) ${ }^{20}$.

Del mismo modo, las comisiones y organizaciones de ayuda también clasificaron los campos de refugiados en dos tipos: los campos civiles y los campos militares. Los campos civiles estuvieron presentes en toda la geografía francesa y fueron destinados a acoger mujeres, niños y ancianos. Estos centros, a menudo denominados también apadrinados o patrocinados (parraines), estuvieron mantenidos por diversos comités internacionales.

civiles cercanos. Correspondencia del inspector departamental de higiene con el prefecto de Ariège (19 de junio de 1937), ADARI, caja 113W/25. Correspondencia del prefecto de Aude

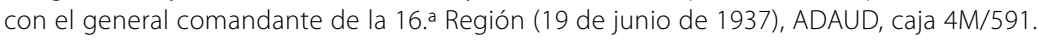

15. Correspondencia del comisario especial de Carcassonne con el prefecto de Aude (2 de abril de 1938), ADAUD, caja 4MD/591.

16. Correspondencia del ministro de la Defensa Nacional y de la Guerra con los generales tenientes de la 16. y y 17. a Región (29 de abril de 1938), ADPO, caja 1287W/1.

17. La répartition des réfugiés dans les départements. Le Temps, 31 enero 1939.

18. Discurso de Albert Sarraut en la Cámara de los Diputados (15 de marzo de 1939), ADPO, caja $31 \mathrm{~W} / 274$.

19. Simón Porolli, Paula. Por los caminos de la palabra. Exilio republicano español y campos de concentración franceses: una historia del testimonio. Universidad Autónoma de Barcelona; 2011, p. 68.

20. Nota del Ministerio del Interior sobre los campos (18 de marzo de 1941), ANP, caja F7/15087. 
La recepción y el alojamiento de la población civil (mujeres, niños y ancianos) fue diferente a la de los militares de la «Retirada» de 1939. Por ello, consideramos que debemos hacer una diferenciación historiográfica entre los campos de concentración, donde se recibió en su mayor medida población militar, y los centros de alojamiento, donde fue recibida la población civil ${ }^{21}$.

\section{La llegada de las organizaciones de ayuda a los centros de alojamiento}

A medida que fueron llegando mujeres, niños y ancianos a los puntos fronterizos, desde finales de enero de 1939 fueron reconducidos hacia el interior de Francia. En 1937 se decretaron 45 departamentos autorizados a recibir refugiados españoles ${ }^{22}$, pero el éxodo de 1939 desbordó dicha normativa, y los más de 210 mil refugiados civiles fueron alojados en 79 departamentos franceses ${ }^{23}$.

Una de las organizaciones de ayuda más activas fue la Commission d'Aide aux Enfants Espagnols Refugies en France (CAEERF). Ésta fue creada por la Commission Internationale d'Aide aux Enfants Evacués en Espagne (CIAEEE) y del Friend Service Council (FSC). Originalmente la CAEERF estaba compuesta por Malaterre-Sellier, Chevalley, Monbrison y Rourdet ${ }^{24}$. El 17 de febrero de 1939, el Ministerio del Interior acreditó a dicha Comisión para visitar a todos los centros de alojamiento. A la vez la CAEERF mantuvo un constante contacto con los Ministerios del Interior, Salud Pública y Finanzas franceses ${ }^{25}$.

La primera tarea de la CAEERF fue nombrar delegados, en su mayoría mujeres, para que se pusieran en contacto con las autoridades departamentales francesas. Dicho comité de delegados estuvo compuesto por educadores, médicos, enfermeras y personal voluntario. Algunos de los voluntarios ya

21. Esta clasificación también ha sido utilizada por otros investigadores como Parello, Vincent. Des camps de réfugiés espagnols de la guerre civile dans l'Hérault. Mélanges de la Casa de Velázquez. 2011; 41 (1): 233-249. Rafaneau-Boj, n. 4.

22. Dreyfus-Armand, n. 1, p. 39.

23. Libro resumen de la Conferencia Internacional de Ayuda a los Refugiados Españoles (París, 15 y 16 de julio de 1939), ANF, caja 20010221/9. Informes diversos de la Commission d'Aide aux Enfants Espagnols Refugies en France (1939), ANF, caja 20010221/1. Rubio, n. 14, p. 210.

24. En los informes no se detallaba el nombre de pila de los delegados o los voluntarios, se ha detallado en el texto cuando ha sido posible conocerlo.

25. Informes diversos, n. 23. 
habían trabajado durante la guerra civil y hablaban español. Por parte de la CAERRF los delegados fueron: Norma Jacob, Katherine Cooper, Lucy Palser, Jacinta Landa y Domingo Ricart. Por parte de la CIAEEE hemos podido identificar la labor de Kershner e Ibargoyen. Además, se sumaron tres voluntarios españoles: Mahou, San Martín y Carmen Mesa; y tres franceses: Morbison, Lesage y Assathiany. También colaboraron en algunas visitas el Comite Neutre Suisse, el Comite Catholique Belgue, la Union Internationale de Secours aux Enfants y la fundación Save the Children ${ }^{26}$.

Los 14 delegados, organizados en ocho equipos fueron los responsables de visitar los más de 1.500 centros, desde marzo a junio de $1939^{27}$. Tras cada visita, emitían informes a la secretaria de la Comisión. Unos informes bastante exhaustivos que explicaban la situación sociosanitaria de los refugiados. Una tarea que se mantuvo de forma constante durante meses y que fue agotadora, como podemos leer en uno de los informes de la delegada Katharine Cooper:

«Por favor perdona esta carta mal escrita. Salimos a las 8:30 de la mañana. Hemos conducido 130 millas [ $209 \mathrm{Km}$ ], hemos visto 6 centros y hemos vuelto a las 7:30 de la tarde y estoy más muerta que viva» ${ }^{28}$.

Entre las funciones desarrolladas por la CAEERF cabe destacar el envío de ropa, material sanitario, escolar y algunos fondos económicos. Los voluntarios que visitaban los centros identificaban aquellos refugiados que no podían volver a España sin peligro de ser represaliados, facilitaban la reagrupación familiar, participaban en la mejora de la educación de los niños y estudiaban las posibilidades de cada departamento para la creación o mejora de los centros ${ }^{29}$.

Las primeras visitas estuvieron encaminadas a establecer un estado de la cuestión y anotar las necesidades materiales de los refugiados. Los delegados del comité obtenían la información de los alcaldes, los comités de acogida y los propios refugiados. En algunas ocasiones también preguntaban a médi-

\footnotetext{
26. Informes diversos, n. 23.

27. Existe un listado de centros de alojamiento de julio de 1939 en ANF, 20010221/9.

28. «Please excuse this badly typed letter. We went out at 8,30 A.M. have driven 130 miles [209 km], seen 6 centers and got back at 7,030 P.M. and I am rather more dead than alive» [Traducción propia]. Informes diversos, n. 23.

29. Informes diversos, n. 23.
} 
cos, enfermeras y maestras que atendían a los niños. A continuación, los delegados emitían sus informes y los enviaban a la comisión, los alcaldes y los departamentos para pasar el siguiente paso, que era enviar materiales de primera necesidad allí donde fueran necesarios. Para abril de 1939, gracias la intermediación de la CAEERF, se enviaron más de 1.500 camas y 3.500 sábanas a 25 centros. Los delegados de la CAEERF también repartieron más de 3.600 pares de zapatillas en 18 centros, circunstancia que hizo que muchas delegadas recibieran el apodo de "las señoras de los zapatos" por parte de los refugiados ${ }^{30}$.

\section{Las condiciones de vida}

Desde finales de enero de 1939 los municipios franceses comenzaron a recibir los primeros refugiados de la «Retirada». Algunos alcaldes ya tenían experiencia con esta situación pues llevaban años alojando a españoles que huían de la Guerra Civil. Para otros, se convirtió en un auténtico reto debido a las limitaciones logísticas y la controversia social que suscitaba la llegada de los refugiados entre la población local. De la noche a la mañana, se acondicionaron cientos de establecimientos para acoger a los refugiados. Además, en tanto que la vacunación sistemática de los niños contra la viruela no se pudo llevar a cabo en su totalidad en la frontera, también se acabó delegando dicha función a los centros de alojamiento ${ }^{31}$. Tampoco se pudo cumplir con las medidas de desparasitación y cuarentena, debido principalmente a la ausencia de duchas y el hacinamiento al que se vieron abocados muchos de los refugiados ${ }^{32}$.

30. Reunión de la Commission d'Aide aux Enfants Espagnols Refugies en France (21 de abril de 1939), ANF, caja 20010221/1. Boletín número 37 del Friends Service Council (2 de agosto de 1939), ANF, caja 20010221/9.

31. Les secours aux réfugiés espagnols. Bulletin de l'Union des Femmes de France. 1939; 20 (2): 34-35.

32. Circular del ministro de Sanidad Pública (17 de febrero de 1939), ADAUD, caja 4M/603; ADTA, cajas 4M/18-12 y 4M18/12; ADTG, caja 4M/4. Correspondencia del prefecto de Aude con ministro de Sanidad Pública (10 de febrero de 1939), ADAUD, caja 4M/603. 
Tabla 2

Densidad de refugiados (número de refugiados y media por centro) por zonas (deparments) en Francia ${ }^{33}$

\begin{tabular}{|c|c|c|c|}
\hline $\begin{array}{l}\text { Departments con mayor } \\
\text { densidad de refugiados }\end{array}$ & N. ${ }^{\circ}$ de centros & $N .^{\circ}$ de refugiados & $\begin{array}{c}\text { Media de refugiados } \\
\text { por centro }\end{array}$ \\
\hline Isère & 1 & 2.500 & $2.500,0$ \\
\hline Ille-et-Vilains & 4 & 4.000 & $1.000,0$ \\
\hline Orne & 4 & 1.773 & 443,3 \\
\hline Manche & 6 & 1.475 & 245,8 \\
\hline Deux-Sèvres & 10 & 2.377 & 237,7 \\
\hline Landes & 14 & 3.014 & 215,3 \\
\hline Charente & 15 & 3.140 & 209,3 \\
\hline Seine-et-Marne & 11 & 2.200 & 200,0 \\
\hline Ardèche & 11 & 1.899 & 172,6 \\
\hline Loire & 9 & 1.547 & 171,9 \\
\hline $\begin{array}{l}\text { Departments con menor } \\
\text { densidad de refugiados }\end{array}$ & N. ${ }^{\circ}$ de centros & $N .^{\circ}$ de refugiados & $\begin{array}{c}\text { Media de refugiados } \\
\text { por centro }\end{array}$ \\
\hline Allier & 47 & 677 & 14,4 \\
\hline Sarthe & 30 & 454 & 15,1 \\
\hline Haute-Saône & 59 & 1.100 & 18,6 \\
\hline Bouches-du-Rhône & 4 & 90 & 22,5 \\
\hline Ariège & 17 & 385 & 22,6 \\
\hline Haute-Vienne & 47 & 1.068 & 22,7 \\
\hline Gard & 62 & 1.584 & 25,5 \\
\hline Yonne & 34 & 880 & 25,9 \\
\hline Lot & 86 & 2.297 & 26,7 \\
\hline Cotes-du-Nord & 69 & 1.985 & 28,8 \\
\hline
\end{tabular}

En mayo de 1939, la CAEERF contabilizó 1.557 centros de alojamiento en Francia. Muchos de los niños fueron enviados directamente a las casas de colonias vacacionales francesas. Esta medida resultó temporal ya que,

33. Elaboración propia, según datos ofrecidos por el Friends Service Council. Boletín n. 37 del Friends Service Council, 2 Ago 1939, ANF, caja 20010221/9. 
con la llegada del verano y las vacaciones escolares en Francia, se produjo el desalojo de los niños y las instalaciones volvieron a su cometido original. De igual manera, en muchas ciudades se acondicionaron grandes espacios municipales como salas de fiestas, gimnasios, cines, teatros, mercados, almacenes o pabellones deportivos. Lamentablemente, estos espacios hubieron requerido una mayor inversión económica y esfuerzo humano para habilitarlos. Muestra de lo dicho es el informe realizado por las delegadas de la CAEERF tras su visita al estadio municipal de Niort (Deux-Sèvres):

«El campo del Estadio Municipal es bastante deplorable. No habría posible solución si no se obliga a partir a esas quinientas personas a otro departamento. El prefecto de Deux-Sèvres se había ofrecido para alojar 1.200 y ha recibido 2.500 [refugiados]; parece convencido que no puede encontrar nada mejor para los que están en el Estadio. Duermen todos dentro de un enorme barracón donde las corrientes de aire y la lluvia pasan entre las planchas mal unidas. Con lonas en el exterior habría menos corrientes de aire pero entonces habría una total oscuridad y esas 500 personas no tendrían ventilación. Para lavar la ropa y los platos para todo el mundo hay dos tinajas de madera. Armarios, cuatro en estado indescriptible. Se cocina casi al aire libre y los días de lluvia se inundan las cocinas. Los colchones de los refugiados se extienden sobre planchas que reposan directamente sobre el suelo pantanoso. (...) Los refugiados parecen atontados y dicen que nadie se ocupa de ellos (...). La señorita Rèchard es casi la única persona que se interesa de esas pobres gentes y ella no sabe qué hacer. Algunos municipios se han ofrecido para alojarlos, pero las autoridades de Niort se han negado diciendo que será un peligro para la salud pública» ${ }^{34}$.

34. «Le camp du Stade Municipal est tout à fait déplorable. II n'y aurait peut-être pas de solution si ce n'est en faisant partir ces cinq cents personnes dans un autre département. Le Préfet des Deux-Sèvres s'était offert pour héberger 1.200 et il en a reçu 2.500 [réfugiés]; il parait convaincu qu'il ne saurait rien trouver de meilleur pour ceux qui sont au Stade. Ils dorment tous dans un énorme baraquement où les courants d'air et la pluie passant entre les planches mal jointes. Avec de bâches à l'extérieur il y aurait moins de courants d'air mais alors ce serait l'obscurité totale et ces 500 personnes n'auraient plus de ventilation. Pour laver le linge et la vaisselle pour tout le monde il y a deux grands baquets en bois. Cabinets, quatre en un état indescriptible. La cuisine se fait presqu'à l'air libre et les jours de pluie les cuisinières sont inondées. Les paillasses des réfugiés sont étendus sur des planches qui eux, reposent directement sur le sol marécageux. (...) Les réfugiés paraissent abrutis et disent que personne ne s'occupe d'eux (...). Mme. Rèchard est presque la seule personne qui s'intéresse à ces pauvres gens et elle ne sait que faire. Quelques communes se sont offerts pour les loger, mais les autorités de Niort ont refusé en disant que ce serait un danger pour la santé publique» [Traducción propia]. Informes de Lucy Palser, Jacinta Landa y David Luscomb de las visitas a los refugiados civiles alojados en los departamentos de Ariège, Basses-Pyrénées, Charente, Deux-Sèvres, Dordogne, Landes, Loir-et-Cher, Loiret y Oise (Niort, Deux-Sèvres, junio de 1939) ANF, caja 20010221/1. 
Como vemos, estos grandes centros de alojamiento, al igual que los campos de concentración del sur de Francia, se caracterizaron por el hacinamiento, la falta de abrigo, las inundaciones recurrentes y la vida en cautividad de los refugiados que albergaban. No pudo ser de otra manera pues, de la noche a la mañana, las prefecturas vieron como la llegada de refugiados superó la cifra de personas que estaban dispuestos a recibir, por lo que tuvieron que dar una respuesta inminente.

También se acondicionaron viejos establecimientos que habían permanecido cerrados durante años como por ejemplo viejas prisiones, hospitales, sanatorios, escuelas u hoteles. Entre todos ellos, las prisiones fueron los lugares más denunciados tanto por los informes de la CAEERF como por los médicos locales ${ }^{35}$. En primer lugar, se señalaba que las instalaciones penitenciarias estaban abandonadas y en pésimas condiciones para ser habitadas, pero además se argumentaba que eran espacios tristes y poco apropiados para albergar a la población infantil. Así se refleja en el siguiente fragmento de informe:

«Aquí, el estado sanitario choca terriblemente. Más de trescientos refugiados están alojados dentro de una prisión en desuso. (...) Los refugiados se quejan sobre todo de estar constantemente enfermos dentro de ese pequeño espacio, detrás de los muros que no dejan ver nada del mundo exterior. Piden permiso para salir. (...) Se alude siempre a la salud pública, pero no se molestan en desatascar los baños de la prisión, lo que provocaría muy fácilmente una epidemia. Los niños no reciben clases, deberían ir a la escuela del pueblo, pero no dejan salir a nadie de la prisión» ${ }^{36}$.

Ante la urgencia, cualquier espacio fue empleado para el alojamiento de la población refugiada. Los ayuntamientos pusieron a disposición antiguas casas parroquiales, orfanatos, hospitales u hospicios regentados por monjas. En esta línea, fueron muy comunes los alojamientos en instituciones religio-

35. Parello, n. 6, p. 240.

36. «lci, l'état sanitaire choque terriblement. Plus de trois cents réfugiés sont hébergés dans une prison désaffectée. (...) Les réfugiés se plaignent surtout d'être constamment enfermés dans ce petit espace, derrière ces murs qui ne laissent rien voir du monde extérieur. Ils demandent la permission de sortir. (...) On invoque toujours la santé publique, mais on ne se donne pas la peine de déboucher les cabinets à l'intérieur de la prison, chose qui provoquerait très facilement une épidémie. Les enfants ne font pas la classe, ils auraient peu aller à l'école de la ville mais on ne laisse sortir personne de la prison» [Traducción propia]. Informes de Lucy Palser, Jacinta Landa y David Luscomb, n. 34. 
sas. En el caso particular de los conventos de clausura incluso se obligó a las refugiadas a adoptar la vida conventual. En el convento Buen Pastor de Le Mans (Sarthe), las mujeres manifestaron un estado psicológico lamentable, según apuntaron las delegadas del CAEERF. Llevaban más de un mes sin ningún tipo de comunicación con el exterior y tuvieron que adoptar la misma vida cuaresmal que las monjas del convento. Desde el CAEERF solicitaron que las refugiadas fueran trasladadas a otro centro ${ }^{37}$.

Como hemos visto, los centros de alojamiento del ámbito urbano se caracterizaron por el hacinamiento, la insalubridad y el trato deshumano. Como contrapunto, encontramos el ámbito rural, donde en la mayoría de los casos los refugiados fueron mejor recibidos y vivieron en mejores condiciones de vida. De hecho, la población rural se volcó con la causa de los refugiados aportando gran cantidad de ropa, comida y, sobre todo, poniendo a disposición de las familias españolas sus casas y apartamentos, algunos en el mismo municipio o en pedanías cercanas. A cambio, en la mayoría de los casos, las españolas cocinaron y ayudaron en las tareas domésticas a quienes con tanta generosidad les había acogido.

Algunos huérfanos fueron integrados en casas de familias francesas, donde se cubrieron todas sus necesidades. Este fenómeno no sólo ocurrió en casas humildes. De igual forma algunas autoridades municipales siguieron el ejemplo de sus paisanos. Por ejemplo, los alcaldes de Castillon (Ariège), Le Fougaz (Ariège) y Masseube (Gers) alojaron en sus domicilios refugiados españoles $^{38}$. Otros alcaldes, como el de Lievin (Pas-de-Calais), visitaron diariamente a los refugiados. Justamente este último municipio guardaba en su memoria lo que su región sufrió durante la Gran Guerra ${ }^{39}$. Desafortunadamente no todas las experiencias fueran positivas y hubo localidades que no se comprometieron tanto con la atención de los recién llegados. Así puede advertirse en el siguiente fragmento de un informe de la CAEERF que hace alusión al alojamiento de los refugiados en el municipio de Marciac (Gers):

37. Informes de la Office International pour l'Enfance a los refugiados civiles alojados en los departamentos de Eure, Oise y Sarthe (Bar-Sur-Seine, Aube, marzo de 1939), ANF, caja 20010221/1.

38. Informes de Katharine Cooper, alias Kanty, de los refugiados civiles alojados en los departamentos de Lot, Lot-et-Garonne, Gers, Drôme, Ariège, Aveyron, Haute-Garonne, Lozère, Tarn-et-Garonne y Tarn, abril de 1939, ANF, 20010221/1.

39. Informes de Kershnery e Ibargoyen sobre las visitas a los refugiados civiles alojados en los departamentos de Aube, Haute-Marne, Marne y Pas-de-Calais (1939), ANF, caja 20010221/1. 
«El ambiente fue envenenado por el alcalde del pueblo, quien estaba constantemente intentando ejercer presión sobre las mujeres para que volvieran a España, una perspectiva que les inundaba de miedo. (...) El alcalde trató de mantener que el miedo de las mujeres era causado por comunistas franceses "agitadores" locales con los que habían estado en contacto, él mismo estaba bastante convencido de que Franco era un perfecto "Caballero Cristiano" ${ }^{40}$.

El caso del municipio de Marciac es un ejemplo más de lo que supuso la política improvisada del gobierno francés de Daladier. Las prefecturas y los ayuntamientos aplicaron una política arbitraria de repatriación en sus municipios, lo que fomentó la desconfianza y el miedo de los refugiados hacia las autoridades locales ${ }^{41}$.

A los refugiados con peor suerte les esperaban espacios menos confortables, algunos inhumanos, como: granjas, establos, molinos, fábricas, garajes, cuevas y viejos castillos. Existió un molino en ruinas donde vivieron hacinados 20 mujeres, 10 hombres y 17 niños. Incluso algunos refugiados fueron integrados dentro de los municipios, otros fueron apartados en colinas o montañas, lugares donde apenas recibieron visitas por parte de autoridades o la población autóctona. Aun así, sorprende ver cómo los españoles se mostraron agradecidos y satisfechos con lo que los franceses les ofrecían ${ }^{42}$.

En cualquier caso, la situación era complicada para todos ya que, incluso antes de la llegada de los españoles, la población local vivía en circunstancias muy humildes. Desgraciadamente los exiliados tuvieron que adaptarse a unas condiciones difíciles y que, en algunos casos, incluso empeoraron tras su llegada. Ciertamente, los refugiados multiplicaron ampliamente el número de residentes en algunos municipios franceses, como es el caso de Monbert (Gers), un pueblo donde sólo vivían 3 franceses y 40 españoles $^{43}$.

40. «The atmosphere was poisoned by the local mayor, who was perpetually trying to exert pressure on the women to make them go back to Spain, a prospect which fills most of the with fear". (...) The mayor tried to maintain that the fear of the women was caused by local French Communist "agitators" with whom they had come into contact, he himself being quite convinced that Franco was a perfect "Christian gentleman" [Traducción propia]. Informes de Gilbert Lesage y Luis San-Martin de las visitas a los refugiados civiles alojados en los departamentos de Cantal, Yonne, Nièvre, Allier y Haute-Loire (Bort-les-Orgues, Corrèze, abril de 1939), ANF, 20010221/1.

41. Léger, n. 6.

42. Informes de Lucy Palser, Jacinta Landa y David Luscom, n. 34.

43. Informes de Katharine Cooper, n. 38. 


\section{La presión psicológica y el aislamiento social}

Las delegadas y los voluntarios de las asociaciones de ayuda no sólo reflejaron en sus informes el deficiente estado físico de las refugiadas, también plasmaron el pésimo estado psicológico observado durante sus visitas. Por ejemplo, Kanty Cooper, una de las responsables de la comisión de visitas a los centros, identificó a unas mujeres atormentadas ante la posibilidad real de que las separaran de sus hijos para enviarlos a Bélgica y que nunca los volvieran a ver. Cooper cuenta que se daban situaciones en las que las mujeres eran avisadas de la marcha de sus hijos apenas unas horas antes y eran encerradas bajo llave hasta el momento de la partida ${ }^{44}$.

En Revel (Haute-Garonne), Cooper denunció en un informe que 220 refugiadas explicaban haber sido engañadas. Se les había dicho que iban a ser trasladados a un nuevo centro en Pau (Pyrénées-Atlantiques) cuando en realidad pretendían mandarlas de regreso a España. Las propias refugiadas narraban como en un afán por representar "normalidad" habían visto subir al tren a viajeros franceses pero que en realidad era una pantomima pues inmediatamente estos supuestos viajeros descendían por el otro extremo del tren. Además, advertían que, en un momento dado del viaje, mientras el tren hacía una parada, se les había dicho que descendieran de los vagones para tomar un café y hacer trasbordo. Algunos, los más desafortunados, siguiendo las indicaciones bajaron del tren, sin darse cuenta de que el tren estaba justo en la frontera. Otros, por suerte, se mantuvieron en los vagones negándose a salir del tren al ver una bandera franquista ${ }^{45}$.

La realidad de las repatriaciones forzadas fue denunciada por Henri Wallon, profesor y presidente de la Office International pour l'Enfance (OIE). Éste publicó un artículo que tuvo gran repercusión en la prensa. En él se reflejó una realidad incómoda para las autoridades políticas francesas, tal y como puede leerse en el siguiente extracto:

«El Gobierno Francés alega verse obligado a deshacerse de los niños porque no puede cubrir sus gastos mediante el oro español depositado en Francia. (...) ¿Deshacerse de los niños para evitar una carga? Razonar así sería empujar una sociedad al suicidio. Reclamar los niños, es reclamar la riqueza más preciada.

44. Informes de Katharine Cooper, n. 38.

45. Informes de Katharine Cooper, n. 38. 
Liberarlos es librar esa riqueza. Librarlos en contra de su voluntad, contra la de sus padres es el acto más vil» ${ }^{46}$.

Las mujeres sufrieron una constante presión por parte de las autoridades galas para que volvieran a España. Muchas de ellas ya habían padecido la represión franquista durante la guerra civil y sentían auténtico pánico hacia todo lo que el régimen de Franco simbolizaba. El temor y la desconfianza fueron tales, que las refugiadas llegaron a pensar que los propios delegados de la CAEERF eran franquistas disfrazados que venían para engañarlas y devolverlas a España ${ }^{47}$.

Ese miedo no era infundado. En caso de regresar a España, las profesionales sanitarias que habían trabajado y colaborado con el ejército republicano se tendrían que enfrentar a largas penas de prisión y/o de muerte ${ }^{48}$. Enfermeras como María Cruz Cerrato Rodríguez, de 22 años de edad y original de Torquemada (Palencia), comunicó a la policía que no podía volver a España porque sería represaliada ${ }^{49}$. María Cruz había entrado por Le Perthus el 4 de febrero y acabó alojada en Lavaur (Tarn) donde se dirigió a la policía para solicitar que no la repatriaran. Ya en 1936 había huido a Francia siendo repatriada a Cataluña en septiembre de 1937. A partir de entonces trabajó en el hospital militar de Barcelona hasta que, en enero de 1939, decide exiliarse definitivamente a Francia tras su participación activa en la evacuación de heridos de Le-Perthus a Perpignan.

A la presión psicológica anteriormente citada se unió el aislamiento social. Primeramente, estaba la dificultad del idioma. Excepcionalmente había alguna refugiada que hablaba francés o alguien del pueblo que hablaba espa-

46. Le Gouvernement Français se prétend obligé de se défaire des enfants parce qu'il ne peut plus se couvrir de ses dépenses à l'aide de l'or espagnol qui se trouvait déposé en France. (...) Se débarrasser des enfants pour éviter une charge? Raisonner ainsi ce serait pousser une société au suicide. Réclamer des enfants, c'est réclamer la richesse la plus précieuse. Les livrer c'est livrer cette richesse. Les livrer contre leur gré, contre celui de leurs parents c'est le trafic le plus immonde, c'est l'acte le plus vil» [Traducción propia]. Artículo de Henri Wallon: Ne livrez pas des enfants que leurs parents ont voulu sauver (9 de agosto de 1939), ANF, caja 20010221/9.

47. Informes de Lucy Palser, Jacinta Landa y David Luscomb, n. 34. El fenómeno de la represión franquista en el exilio ha sido analizado por Guixé Coromines, Jordi. La república perseguida. Exilio y represión en la Francia de Franco, 1937-1951. Valencia: Universitat de València; 2012.

48. Mirón-González, Rubén. La enfermería profesional en España: Origen, avance y represión (S.XIX$X X)$. En: Morrone, Beatriz. Al servicio de las ideas. La enfermería en los procesos populares de liberación en Iberoamérica. Mar del Plata: Suárez; 2013, p. 235-283.

49. Correspondencia del comisario Lavaur de Tarn con el prefecto de Tarn (20 de febrero de 1939), ADTA, caja 4MD/18-27. 
ñol, especialmente maestras rurales ${ }^{50}$. Además, el interés y la desconfianza que despertaban las recién llegadas hizo que algunas españolas se sintieron intimidadas al ver cómo la población rural francesa fijaba sus miradas sobre ellas. Hasta tal punto se dio esa situación de celo que, en algunos pueblos, la iglesia francesa llegó a impedir la asistencia a misa a las refugiadas católicas ${ }^{51}$.

Por lo general a las refugiadas también se les negó trabajar. Por un lado, tuvieron dificultades para acceder a los trabajos no cualificados pues se las percibía como una competencia directa respecto a la mano de obra francesa, y, por otro lado, las más preparadas tampoco pudieron acceder a puestos cualificados al no ser reconocidos sus títulos académicos españoles. Las exiliadas que tuvieron más suerte llegaron a encontrar algún trabajo en tiendas locales, pero al poco tiempo se les prohibió continuar con el mismo.

Tras analizar los informes de las delegadas se han podido constatar que el departamento de Gers fue uno de los pocos que posibilitó que las refugiadas pudieran trabajar de forma remunerada. En pueblos como Cazaubon, las mujeres tejían calcetines a cambio de 3,50 francos por par, pero lo más común era el trabajo en la limpieza doméstica. En algunos trabajos los hombres ganaban jornales de 10 a 11 francos diarios. Incluso, en ayuntamientos como el de Saint-Sulpice (Tarn) el alcalde entregaba directamente a los españoles el dinero que el municipio recibía en concepto de ayudas a los refugiados para que fueran lo más independientes posibles. Estas cifras variaban de forma arbitraria de un departamento a otro. Por supuesto, en otros pueblos no se les pagaba, alegando que ya se les daba de comer y alojamiento ${ }^{52}$.

\section{La asistencia sanitaria}

Al llegar a los centros de alojamiento, los refugiados fueron sometidos a una exploración física con el objetivo de descartar cualquier tipo de enfermedad venérea o parasitaria. En algunos municipios estos reconocimientos se realizaron en grupo, mezclando a hombres, mujeres y niños, y en presencia de gendarmes y otras personas. Esta situación vivida es recordada por algunas refugiadas como humillante y en contra de su dignidad ${ }^{53}$.

\footnotetext{
50. Informes de Katharine Cooper, n. 38.

51. Informes de Gilbert Lesage y Luis San-Martin, n. 40.

52. Informes de Katharine Cooper, n. 38.

53. Maugendre, n. 10, pp. 94-95.
} 
La mayoría de los niños y mujeres dormían en camastros de paja sobre el suelo frío y húmedo en condiciones de hacinamiento. Esto hizo que aparecieran frecuentemente brotes epidémicos provocando oleadas de muertes. El único modo de frenar estos factores y contagios fue que los recién llegados vivieran en casas individuales, algo prácticamente imposible porque, como ya se ha advertido anteriormente, la mayoría de los centros de alojamiento fueron colectivos ${ }^{54}$. Como puede verse en el siguiente fragmento de informe, en Tarnos (Landes) convivieron 76 niños y 74 adultos en una colonia de vacaciones que estaba sin finalizar, donde las condiciones de salubridad fueron deplorables:

«Algo muy raro había aquí. Al menos hay veinte niños enfermos y seis han muerto, probablemente de sarampión. Tenían dos casos de difteria y bastantes bronquitis y neumonía. Dos enfermeras fueron enviadas desde Labenne ayer, pero con todo el mundo durmiendo en las mismas habitaciones y sin lugar para el aislamiento la epidemia no tiene pinta de disminuir. El médico visita cada día. No hay ninguna persona competente al cargo ${ }^{55}$.

Como norma general, no existía un protocolo asistencial específico para los refugiados civiles (mujeres, niños y ancianos). En el mejor de los casos, los niños eran atendidos diariamente por médicos del Servicio Departamental de Higiene. Gracias a las visitas del CAEERF, se pudieron identificar aquellos centros que requerían urgentemente un control sanitario riguroso. Un año más tarde, en 1940, en los informes se detecta un incremento de las visitas de enfermeras, médicos y trabajadores sociales a los centros ${ }^{56}$.

Pero en los primeros meses tras el éxodo de 1939 las epidemias siguieron su curso. Para justificar esa situación, los inspectores departamentales de higiene se exculparon alegando que eran enfermedades traídas de España debido a las malas condiciones en las que habían llegado los exiliados. Pero

54. Informe de la Union Internationale Services Enfants de las visitas a los refugiados civiles de Ain y Provence. (20 de marzo de 1939), ANF, caja 20010221/1.

55. "Something has been very wrong here. There are at least twenty of the young children ill and six have just died, probably of measles. They had two cases of diphtheria and have many of bronchitis and pneumonia. Two nurses were sent from Labenne yesterday, but with everyone sleeping in the same rooms and no place for isolation the epidemic does not seem likely to subside. The doctor visited every day. There is no very capable person in charge» [Traducción propia]. Informes de Lucy Palser, Jacinta Landa y David Luscomb, n. 34.

56. Informe sobre gastos de desplazamiento de refugiados en el departamento de Ariège (1 de julio de 1940), ADARI, caja 113W/60. 
lo que no denunciaban las autoridades francesas era las condiciones de hacinamiento e insalubridad en las que vivían las refugiadas ${ }^{57}$.

A pesar de que los principales objetos que pedían los refugiados de los campos era jabón, cepillos y pasta de dientes. La falta de higiene hizo que las enfermedades cutáneas derivaran en infecciones. A eso debe sumarse que las enfermerías en los grandes centros de alojamiento fueron prácticamente inexistentes. En el caso de que se contara con alguna enfermería, éstas carecían de material, viéndose obligadas las enfermeras a derivar los pacientes a los hospitales departamentales. Por otro lado, las propias enfermeras francesas preferían que los enfermos permanecieran en los centros y que no fueran trasladados a los hospitales. En el caso de los hospitales regentados por religiosas, se ponía como excusa una falta de preparación por parte del personal de asistencia, aunque parece que la auténtica razón eran los desencuentros entre las refugiadas y las religiosas francesas, tal como vemos en el siguiente extracto de informe ${ }^{58}$ :

«No son muy bien acogidos [los refugiados]. (...) Existían muchas discrepancias entre monjas y refugiados. En todos los campos que hemos visitado que están al cuidado de monjas hemos encontrado las mismas características. Debe ser la general actitud de las monjas hacia los refugiados (no es tanto lo que dan sino cómo lo dan) eso saca lo peor de aquellos que están bajo su cuidado» ${ }^{59}$.

La naturaleza de las discrepancias entre las religiosas francesas y los refugiados civiles, en su mayoría mujeres, no queda del todo clara en los informes de las delegadas de la CAEERF. Siguiendo la investigación de Eva Léger, todo apunta a que estos desacuerdos podrían tener su origen en el estigma de las «mujeres, extranjeras y "rojas" ${ }^{60}$ que estaba difundiendo la prensa de derechas en Francia, aunque también podría haberse debido a una actitud combativa de las españolas ante las medidas restrictivas que

57. Informe del inspector departamental de higiene de Tarn-et-Garonne (11 de marzo de 1939), ADTG, caja 4M/4.

58. Informes del Comite Neutre Suisse de Secours aux Enfants... ANF, 20010221/1. Informes de la Office International pour l'Enfance, n. 37.

59. «They are not well housed [los refugiados]. (...) There were many grievances from both the nuns and the refugees. In all the camps we have visited that are in the care of nuns we have found the same characteristics. It must be the general attitude of the nuns towards the refugees (it is not so much what they give as how they give it) that calls out the worst in those under their care» [Traducción propia]. Informes de Lucy Palser, Jacinta Landa y David Luscomb, n.34.

60. Léger, n. 6, p. 79. 
estaba llevando a cabo el Estado francés sobre los refugiados. Como vemos, la represión y la imagen social de los refugiados también tuvieron consecuencias en el plano asistencial.

A pesar de los esfuerzos de las organizaciones de ayuda humanitaria, todavía quedaba mucho por hacer en pro de la asistencia sanitaria de los refugiados durante los primeros meses de la «Retirada». En este sentido, fue crucial la Conferencia Internacional de Ayuda a los Refugiados Españoles, celebrada el 15 y 16 de junio de 1939 en París ${ }^{61}$. En esta Conferencia, se fijaron los desafíos sanitarios que debían ser abordados cuanto antes, entre los que destacamos: la necesidad de cumplir con las medidas de cuarentena, la creación de salas de aislamiento, las visitas médicas regulares, la presencia de un médico español durante las consultas, la necesidad de terminar con la campaña de vacunación, agilizar los ingresos hospitalarios, así como asegurar una correcta alimentación. Estos fueron los puntos débiles detectados que habría que mejorar para atender las necesidades de la población refugiada ${ }^{62}$.

\section{Los rincones blancos}

Según datos de la International Commission for Assitance to Child Refugees in Spain (ICACRS), en 1938 el estado nutricional de los niños del bando republicano era alarmante ${ }^{63}$. Esto se vio agravado a medida que la guerra avanzaba y el acceso a alimentos de primera necesidad se vio cada vez más restringido. También el estado nutricional de los niños acogidos en Francia era preocupante. Desgraciadamente en muchos centros donde estaban refugiados también carecían de alimentos básicos como leche, carne o pescado debido a la pobreza de los propios municipios a la que anteriormente hemos hecho referencia. Básicamente, la dieta consistía en judías, lentejas, patatas y pasta. Esta situación hizo que se crearan los «rincones blancos» (coins blancs), una iniciativa promovida por la Office International pour l'Enfance (OIE) y secundada por muchas otras organizaciones.

\footnotetext{
61. Esta Conferencia tuvo como objetivo buscar soluciones a la situación de los españoles en el exilio. Para ello, se montaron seis mesas temáticas que abordaron la realidad de los refugiados desde el punto de vista laboral, material y sanitario.

62. Libro resumen de la Conferencia Internacional de Ayuda a los Refugiados Españoles, n. 23.

63. Memorandum de la International Commission for Assistance to Child Refugees in Spain (24 de septiembre de 1939). ANF, caja 20010221/1.
} 
Los rincones blancos fueron espacios creados dentro de los centros de alojamiento para asegurar la alimentación mínima de la población infantil. Una idea que no era nueva, ya que el gobierno republicano lo puso en marcha en España durante la guerra civil. Con esta iniciativa se aseguraba que los niños recibieran, al menos, un tazón de leche al día en la merienda junto a una chocolatina o un trozo de pan con mermelada, así como fruta fresca o deshidratada. También se distribuyó, bajo indicación médica, aceite de hígado de bacalao u otros complementos nutricionales ${ }^{64}$.

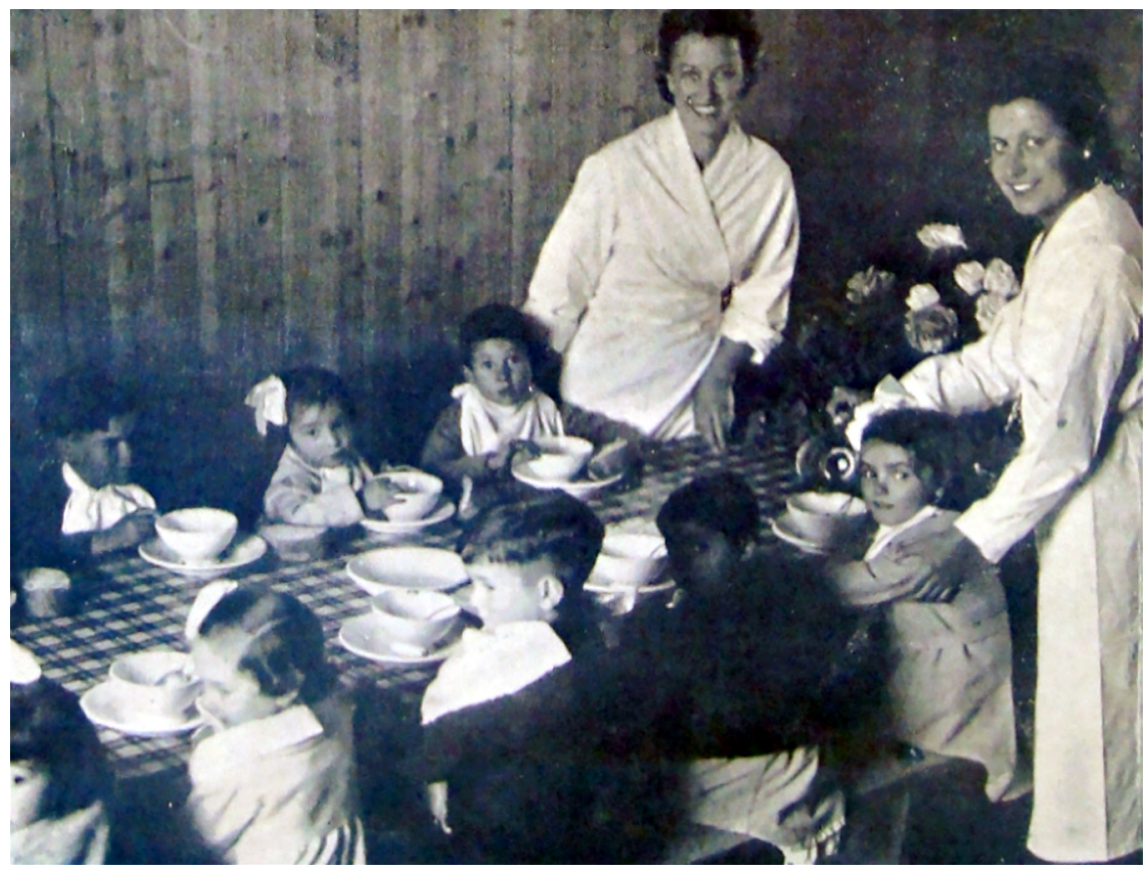

Figura 1: Imagen del interior de un rincón blanco, extraída de un folleto informativo de la Office International pour l'Enfance para la recaudación de donativos (sin fecha) ${ }^{65}$.

64. Office International pour l'Enfance. Coins blancs, ANF, 20010221/9.

65. Office International pour l'Enfance, n. 64. 
Se formaron equipos móviles constituidos por una asistente social francesa y una intérprete española. Mediante camiones, éstas se desplazaron por la geografía francesa con leche en polvo o condensada, azúcar, cacao y aceite de hígado de bacalao; así como todo el material necesario para equipar cocinas y comedores. Una vez llegaban a los centros, formaban a un grupo de tres o cuatro mujeres y nombraban a una responsable, normalmente enfermera o maestra. Ellas se encargaban de preparar y distribuir la leche, así como de la higiene del material y la cocina. Por lo demás, recibían una bata y una breve formación por parte del equipo móvil de cómo organizar el rincón blanco y elaborar la leche ${ }^{66}$.

Con los rincones blancos se garantizó la alimentación de los niños, y, por ende, se mejoró la salubridad en algunos centros de alojamiento. Incluso se procuró que dichos rincones fueran un lugar donde los niños pudieran jugar de forma segura. Del mismo modo se constituyeron como en un punto de educación para la salud en el que las madres, pudieran reunirse y resolver dudas entre ellas. La división de tareas en estos rincones blancos era la siguiente: mientras las responsables del rincón blanco velaban para que reinara la higiene y estaban obligadas a emitir informes mensuales a la OIE. Las refugiadas, eran responsables de asegurar la existencia de los víveres, así como de prever cualquier tipo de escasez con 8 días de antelación. Y finalmente, las enfermeras se encargaban de gestionar los servicios sanitarios que los niños necesitaban ${ }^{67}$.

El objetivo de la OIE era establecer un rincón blanco en cada centro con más de 50 niños. Éste era un objetivo ambicioso, sobre todo si tenemos en cuenta que al menos, 199 centros cumplían este criterio. De abril a julio de 1939 se pusieron en marcha 70 rincones blancos, cubriendo con ellos a más de 8.500 niños. A pesar de los esfuerzos, sólo se logró cubrir el 35\% de los centros que tenían más de 50 niños. En cuatro meses se distribuyeron hasta 18 mil kilogramos de leche, 6 mil kilogramos de azúcar, 10 mil kilogramos de chocolate, 2 mil kilogramos de aceite de hígado de bacalao y 5 mil kilogramos de mermelada; una mercancía valorada en más de un millón de francos ${ }^{68}$.

\footnotetext{
66. Office International pour l'Enfance, n. 64.

67. Office International pour l'Enfance, n. 64.

68. Office International pour l'Enfance, n. 64.
} 


\section{Conclusiones}

Llegados a este punto, podemos confirmar que la población francesa se volcó desde el primer momento para ayudar a los refugiados civiles españoles. Esto ocurrió sobre todo en el contexto rural, donde los refugiados recibieron un trato digno y gozaron de ciertas libertades, a diferencia de algunos contextos urbanos donde vivieron en condiciones penitenciarias e insalubres.

A pesar de la estimable respuesta humanitaria de la población francesa, los refugiados tuvieron que enfrentarse desde el primer momento a dificultades de carácter sociosanitario. Desde el punto de vista social, existió una visión estigmatizada de refugiada como «esposa de marido rojo». Esta imagen, hizo que las refugiadas tuvieran dificultades para relacionarse con población católica, curas o monjas en algunos municipios. Además, el desconocimiento de la lengua francesa y la dificultad para acceder a un trabajo remunerado fueron barreras importantes para lograr una sociabilidad plena. El sentimiento de desconfianza y miedo totalmente justificado de algunas refugiadas a ser engañadas, separadas de sus hijos o repatriadas a España.

Entre las primeras dificultades sanitarias, cabe destacar la falta de infraestructuras en algunos municipios que impidió llevar a cabo una correcta desparasitación y cuarentena de los enfermos. A medio plazo, se dieron otros problemas como la necesidad de establecer visitas sanitarias regulares en los centros de alojamiento, agilizar los ingresos hospitalarios o exigir la presencia de profesionales o intérpretes que actuaran de mediadores entre madres, niños y franceses. Afortunadamente, había enfermeras o médicos entre los refugiados que pudieron colaborar en la asistencia proporcionada por los profesionales sanitarios locales de los municipios de destino.

A pesar de las adversidades, las organizaciones de ayuda humanitarias lograron cambiar las condiciones de vida en los centros de alojamiento franceses durante los primeros meses del exilio. La iniciativa de los rincones blancos es un claro ejemplo de plan de salud en contextos bélicos. Hemos visto como gracias a la organización de recursos materiales, humanos y un poco de formación, en cuatro meses se pudo llevar a cabo un auténtico plan de promoción de la salud y cambiar la suerte de miles de niños y madres.

Por último, cabe destacar la labor de asociaciones y organizaciones de ayuda, como la Commission d'Aide aux Enfants Espagnols Refugies en France o la Office International pour l'Enfance. En el presente artículo se ha documentado como las delegadas y voluntarias de estas organizaciones, mujeres en su mayoría, acudieron al rescate de la población femenina del exilio repu- 
blicano español. Nos encontramos ante un ejemplo más de lo que supuso la creación de redes de ayudas femeninas en el sur de Francia ${ }^{69}$ y cómo las organizaciones de ayuda cumplieron con un rol de liderazgo humanitario ${ }^{70}$.

69. González Canalejo, Carmen. El exilio de las mujeres: trabajo y redes femeninas en los campos del Sur de Francia (1939-1945). En: Ramos Palomo, María Dolores; et al. Mujeres iberoamericanas y derechos humanos. Experiencias feministas, acción política y exilios. Sevilla: Athenaica: 2016, pp. 272-293.

70. Kerén, Celia. Les enfants espagnols réfugiés en France. 1939 ou la crise de la solidarité ouvrière. Les Cahiers du CRH. 2009; 44: 75-89. 